\title{
DOES EMOTIONAL MATURITY HELP TO EXPRESS MORAL BELIEFS? EVALUATING 'MORAL GAMES FOR TEACHING BIOETHICS' AMONGST ROMANIAN AND POLISH STUDENTS
}

\author{
Cristina Iulia Ghenu, ${ }^{1}$ Laura Brad, ${ }^{2}$ Bartosz Płotka ${ }^{3}$
}

\begin{abstract}
The recent development of biotechnology generated a new set of individual and public moral dilemmas gathered under the name of bioethics or biopolitics. These issues are specific because they merge - as nothing else before - moral, private and political spheres. Thus, public awareness of these cases and of any elements that can influence personal bioethical decisions must be stimulated. One of such methods is the academic teaching of bioethics. Since Darryl R.J. Macer defined the latter as "the love of life, reflecting the hope that bioethics may value life in a process involving emotions and rationality" we found interesting to investigate the role of emotional maturity (EM) in solving bioethical dilemmas. The study involved 103 Polish and Romanian students asked first to fill the Friedman's emotional maturity form and then solve chosen exercises based on UNESCO's Moral Games for Teaching Bioethics. The results indicate that a high level of emotional maturity correlates positively with the students' ability to express their moral beliefs for Romanians and negatively for Poles; therefore, the results indicate there is a need to modernize the actual standards for teaching bioethics by enriching them with either emotional or rational components according to the cultural premises.
\end{abstract}

UDC Classification: 172, 177, 378; DOI: http://dx.doi.org/10.12955/cbup.v5.1022

Keywords: emotional maturity, moral beliefs, education, bioethics, biopolitics, Romania, Poland

\section{Introduction}

The recent development of biotechnology generated a new set of individual and public moral dilemmas gathered under the name of bioethics. Because of their contentious and conflict-generating nature they have been politicized which, paradoxically, did not bring solutions to these cases but extended their reach and diffused them throughout societies. Since then, they have become ubiquitous thus having an opinion about them became the inherent element of worldview and conscious democratic participation in a public sphere. In addition, the bioethical (or biopolitical) issues are very specific because they merge - as nothing else before moral, private and political dimensions. This is why public awareness of these cases and of other elements that can influence personal bioethical decisions must be stimulated by social campaigns, public debates, promoting scientific discoveries and education. The latter requires special attention since it is the most engaging and direct method of transferring bioethical knowledge. However, as some discoveries, such as Roache and Clark's (2009) work demonstrate, bioethics involves not only the usual knowledge (rationality) but also intuitions (emotions). This is why we investigate the possible connections between them by answering the research question: how does the level of emotional maturity (EM) correlate with the ability to express moral beliefs (also called bioethics or B)? Based on the literature review and our initial observations we propose and verify the following hypothesis: people with a higher level of emotional maturity express their moral beliefs significantly more clearly than people with a low level of emotional maturity. For the study's purposes, we investigated 53 Romanian and 50 Polish students $(n=103)$ by asking them first to fill the Friedman's emotional maturity questionnaire and then to solve chosen exercises based on UNESCO's Moral Games for Teaching Bioethics. Our results indicate that only Romanian students with high emotional maturity expressed moral beliefs significantly more clearly, while the results for the Polish sample indicate the opposite. Therefore, since bioethics requires both intuitive and rational explanations, our findings indicate there is a need to modernize the actual standards for teaching bioethics by emphasizing the development of emotional component in bioethical reasoning, but only if cultural differences are taken into account.

\section{Theoretical Background}

In this study, we use the newest discoveries of management, human resources, moral philosophy and political science to address the general question: does increasing the public awareness of bioethical issues through education require modernization?

After participating in a number of classes, conferences and seminars on bioethics we have two reasons to believe the answer is positive. First, we discern that many bioethical debates fail in reaching any consensus because the participants suffer from Dworkin's (1986) "semantic sting."

\footnotetext{
${ }^{1}$ Bucharest University of Economic Studies, Faculty of Management, Bucharest, Romania, cristina.ghenu@man.ase.ro

${ }^{2}$ Bucharest University of Economic Studies, Faculty of Finance,Bucharest, Romania, laura.brad@ @in.ase.ro

${ }^{3}$ Nicolaus Copernicus University, Faculty of Political Science and International Studies,Poland, bplotka.umk@gmail.com
} 
They are usually unable to identify the source of disagreement, precisely express their own beliefs and arguments, or even to say what in fact is bioethics. Second, the teaching of bioethics depends mainly on presenting facts and trying to approach them in the most rational way possible, while many debates as well as scholars, such as Kass (1997) or Fukuyama (2002), show that disputants base arguments more often on their moral intuitions than knowledge. If we connect these observations and accept Darryl R.J. Macer's (2008) definition of bioethics - coined as "the love of life" which reflects the expectation that bioethics value life in a way involving rationality and emotions - instead of any normative approach trying to settle what is right in bioethical cases, then we can easily discern that the most important ability in bioethical disputes is to precisely identify and clarify our own moral beliefs, but also facilitates mutual understanding. One thing we cannot omit in Macer's definition is the role of emotions which significantly influences our choices and convictions. Taking it into account, we propose the following logical reasoning: if emotions influence moral hence bioethical beliefs, the disposition of developed emotions and the ability to recognize them influence the ability to precisely identify and clarify these beliefs. Since Salovey and Mayer (1990) denoted emotional intelligence (EI) as the ability to recognize and modify one's emotions in order to achieve intellectual and emotional growth, acknowledging their definition allows us to perceive EI not as a disposition but as an ability. Based on the same idea, Athota et al. (2009) investigated the role of EI in moral reasoning. Although the authors confirmed such a link might exist, they focused mainly on the connection between moral reasoning and personality traits such as: agreeableness, openness, extraversion, neuroticism and conscientiousness. Thus, estimating the correlation between EI and identification of one's moral beliefs based on their study is not sufficient. The same problem concerns the work of Kornilova and Chigrinova. (2014) who also tried to investigate the title connection but their results regarding the clarification of moral beliefs overlap with types of personality and stages of moral development. Other interesting studies were proposed by Parsa (2015) and Fernandez-Berrocal and Extremera. (2005) whose results suggest that moral decision-making might be dependent on the emotions and EI. However, their investigations do not tell us to what extent EI level can impact the clarification of one's moral beliefs thus the quality of moral decisions. We believe the inability of the aforementioned authors to investigate the direct connection between EI and good clarification of moral beliefs is conceptual. It was caused by a fallacy of choosing a narrow concept - emotional intelligence which stands solely for the ability to recognize one's emotions. The solution to the above theoretical problems seems to be focusing on the more general concept which is emotional maturity. This is motivated by the fact that one can have well-developed EI but be emotionally infantile, while the other cannot be emotionally mature without having developed EI. Hence, we recognize EM as the proper factor to correlate with the clarity of moral beliefs and discern a difference between EI and EM - the former is an ability while the latter is a disposition. Based on these observations we support such approach which takes into account EM, as did Arbune et al. (2014), Mărghitan and Craşovan. (2014), Craşovan and Ioviţă (2013) and Moldovan (2014) in their studies including the role and meaning of emotions.

Summarizing, all the aforementioned studies have a similar message: they indicate that EI and EM might play a significant role in moral decision-making. Their disadvantages however, are: choosing 'moral exercises' not necessarily corresponding to EI's and EM's basic assumption of connecting rationality with emotions and not realizing the task of measuring the actual impact of EM level on the ability to clearly express moral beliefs.

\section{Research Methodology}

The research aims to provide evidence on the correlation between emotional maturity level and the ability to express moral beliefs - we call this variable bioethics (B) - for both Romanian and Polish students. In order to conduct the analysis, we used quantitative analysis based on a questionnaire consisting of two parts. The first part of the questionnaire is best described by Craşovan (2013) who considers EM as the element that refers to the Superego strength and Ego objectification, comprising 25 questions of "yes" and "no." While Superego qualities are related to emotional security, a realistic perception about one's self, others and the world, emotional imbalance is caused by Ego weakness, emotional instability and is accompanied by a series of infantile, childish psycho-affective reactions. Each answer of a question has its own score, the final score being obtained by using arithmetic. We used the same scale as proposed by the author in order to quantify the effects of our results.

The second part was built using Moral Games for Teaching Bioethics that provides a number of participatory games in order to teach moral decision-making and expressing moral beliefs (Macer, 2008). Macer did not indicate any method that could be useful for evaluating the Moral Games for Teaching Bioethics, or teaching bioethics in general, in the emotional context. This is why we reconstructed and expanded the set of most 
typical bioethical statements concerning issues such as abortion, euthanasia, human enhancement, organ transplantation, in vitro fertilization, stem cell research, animal rights and general legal and ethical standpoints. The variants "Strongly agree," "Agree," "I have no opinion," "Disagree," "Strongly disagree" were offered for encircling to each 50 proposed statements concerning moral beliefs. A weight was assigned for each question. For 21 statements a one point was assigned and for the others five. These were solely working weights which meant that the numbers 1 and 5 were assigned to define moral beliefs in higher or a lower degree. One can then use other weights, such as A and B, though we used numeric ones to be able to quantify the results.

Since the dependent variable in our study was the ability to clearly express moral beliefs (B) we were not investigating the bioethical worldviews per se, but their intensity. To understand that, we propose to look at the analogy between "to strongly agree vs. to strongly disagree" and "to love with passion vs. to hate with passion". Both love and hatred are qualities like agreeing and disagreeing while passion is their strength indicator. We are interested not into the qualities, but into their intensity.

Students from The Bucharest University of Economic Studies (Romania) and Nicolaus Copernicus University (Poland) filled the questionnaire. The Romanian respondents came from the Faculty of Management, The Faculty of Marketing and the Faculty of Finance, Insurance, Banks and Stock Exchange. The Polish respondents came from the Faculty of Political Science and International Studies. Since they all studied social sciences including management, finance, marketing, political science, internal security and journalism, hence there was a higher probability that they had classes from bioethics. The groups within disciplines were selected randomly. The initial sample consisted of 70 Romanian and 70 Polish students. In order to have a homogenous sample we have excluded the students who took classes, courses and workshops on bioethics or attended bioethical debates. The justification resides on quantifying if knowledge is above or below the emotional level. The exclusion was made on the basis of four questions in the questionnaire about prior contact with bioethics. If just one answer was positive, a questionnaire was eliminated from the further analysis. The final sample comprised 53 Romanian and 50 Polish students.

In order to attain our purpose of research we computed the scores of the questionnaire, we organized the collected data and performed its statistical analysis. The results for the emotional maturity level were related with Craşovan (2013): 0-9.99 (infantile), 10-11.99 (childish emotional reactions), 12-13.99 (psycho-affective reactions), 14-15.99 (slight emotional immaturity), 16-17.99 (tendency for imbalance), 18-19.99 (slight emotional maturity), 20-21.99 (corresponding maturity), 22-23.99 (good maturity), over 24 (perfect emotional maturity). The results for the ability to clearly express moral beliefs ranged between $0-4$. To see if there is an influence of emotional maturity on bioethics score, a regression model was applied. The dependent variable was the bioethics score, while the independent variables were the emotional maturity score together with age and gender. The estimations were conducted in SPSS software. Additional analysis was conducted by providing the influence of gender on both emotional maturity and bioethics score. Considering these aspects, the hypotheses on which the research was conducted were: H1: The higher the degree of emotional maturity is, the higher the clarification of moral beliefs is. $\mathrm{H} 2$ : Women have higher emotional maturity than men.

\section{Results}

The first step was to present the descriptive statistic of the two samples, 53 Romanian students and 50 Polish students. The descriptive statistic is going to be provided for all the variables included in the analysis (Table 1).

Table 1: Descriptive statistic of gender respondents
\begin{tabular}{|c|c|c|c|c|}
\hline & \multicolumn{2}{|c|}{ Romanian sample } & \multicolumn{2}{c|}{ Polish sample } \\
\hline Gender & Frequency & Percent $\%$ & Frequency & Percent $\%$ \\
\hline Male & 16 & 30.2 & 15 & 30.0 \\
\hline Female & 37 & 69.8 & 35 & 70.0 \\
\hline Total & 53 & 100 & 50 & 100 \\
\hline
\end{tabular}
Source: Author

From Table 1 it can be seen that the size of the two samples is approximately the same consisting of 53 Romanian students and 50 Polish students. Also, the gender distribution is similar, with the Romanian sample 
being formed of 16 males and 37 women and the Polish sample consisting of 15 males and 35 women. Hence, the composition of the sample is approximately computed as $30 \%$ male and $70 \%$ women.

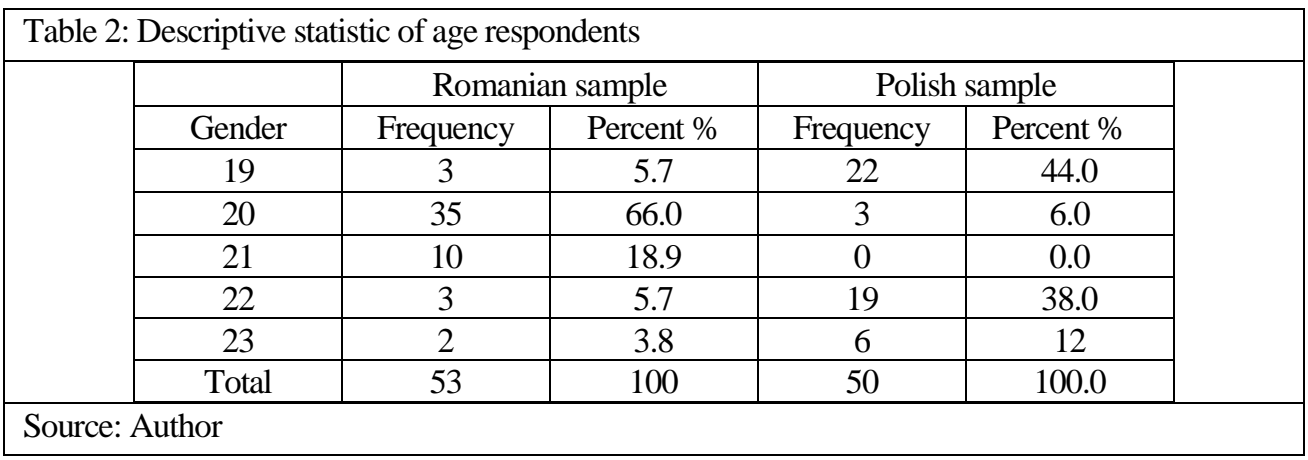

From Table 2 we observed most Romanian students are aged between 20-21 years old, while most Polish students are divided into groups of 19 and 22 years old. Romanian students came from a second year bachelor degree program, while Polish ones came both from bachelor and master degree programs. We consider this structure can influence both EM and B scores. We assume that an increase in age results in an increase in B score. If we consider the average value of this indicator then the average value of Romanian students is 20.36 years old, while the average value of Polish students is 20.68 years old.

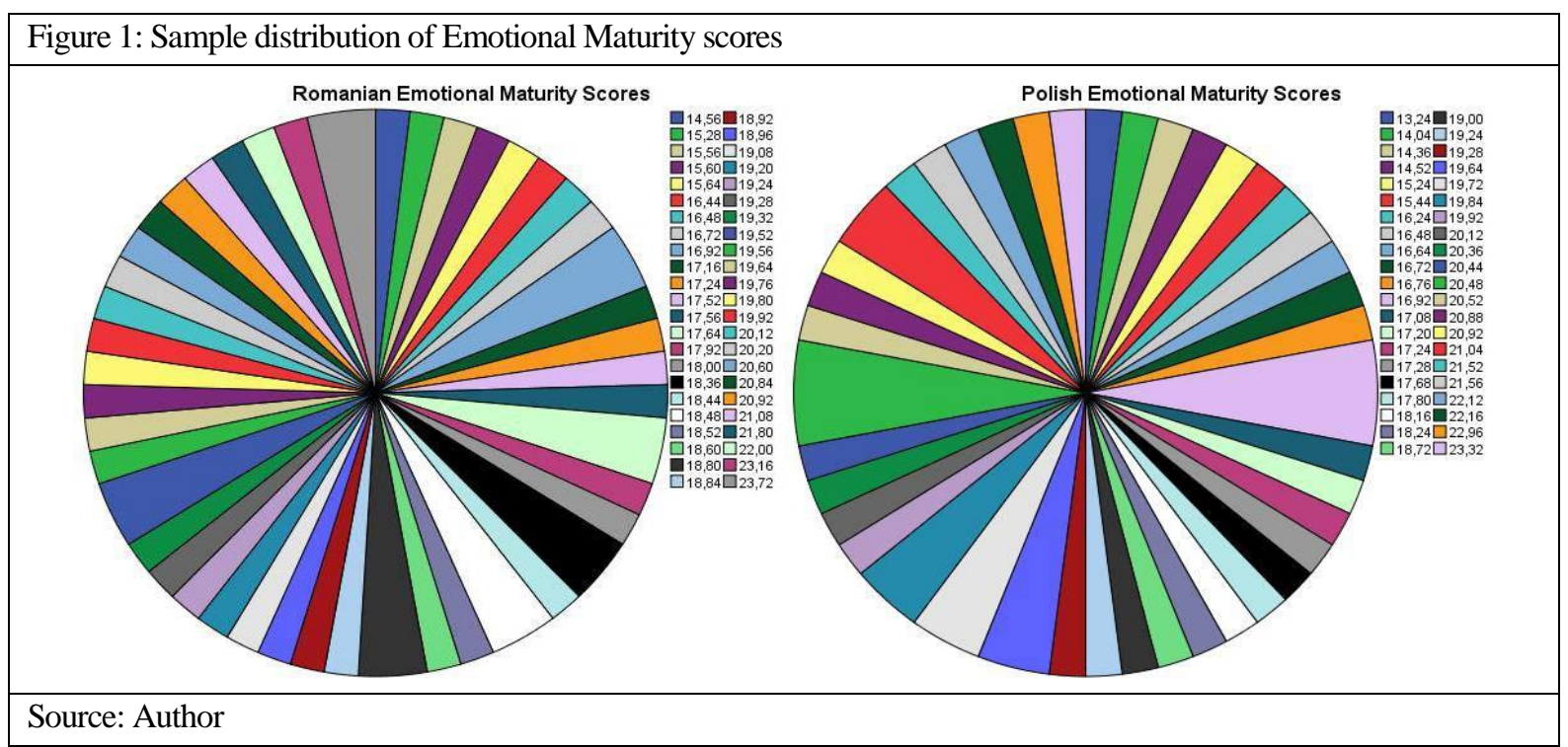

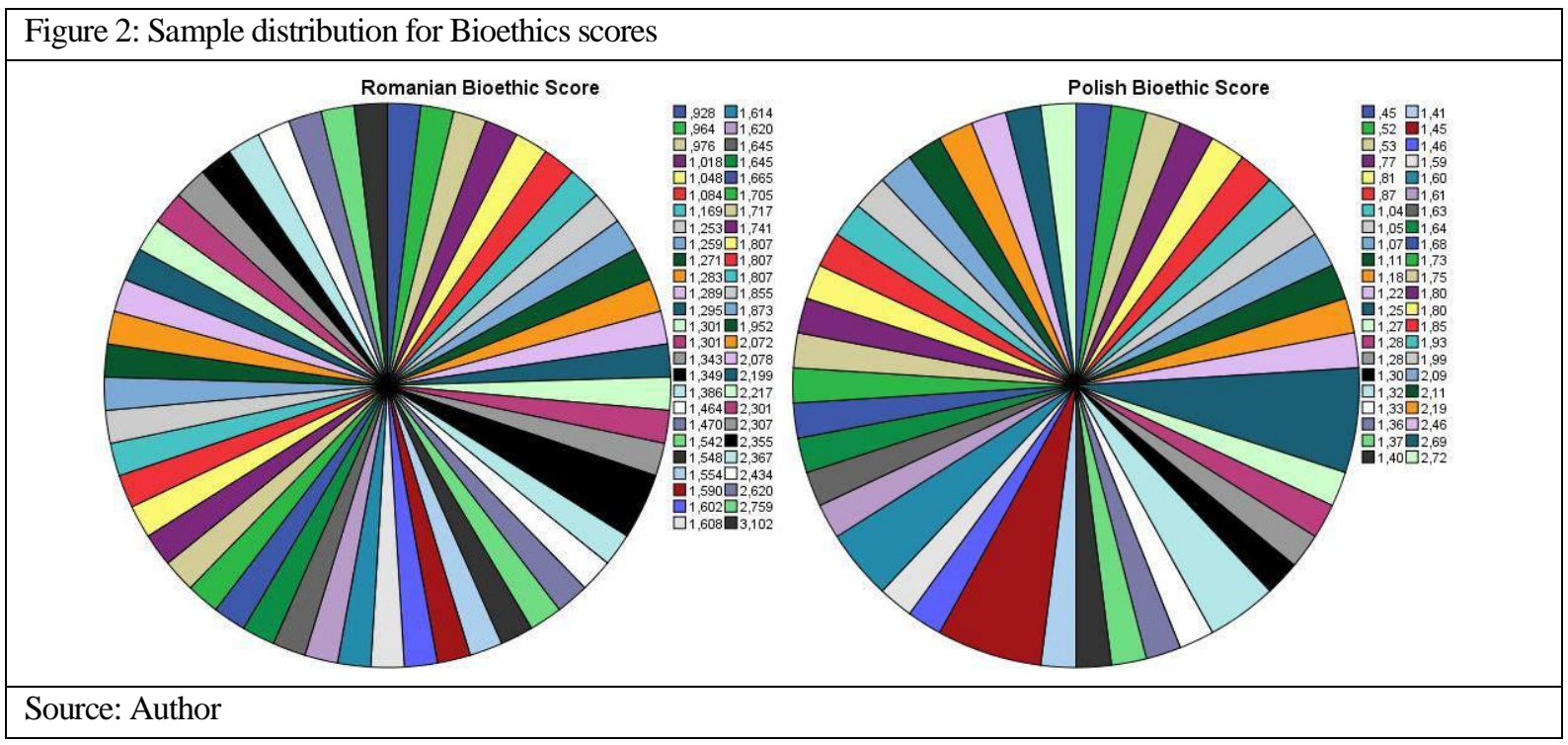


The third variable on which we performed the descriptive statistic was EM. For Romanian students, the min. score was 14.56 and the max. was 23.72. The mean is 18.79 and the median value is 18.81 (with standard deviation of 2.01). For the Polish sample, the min. value was 13.24 and the max. value was 23.32, while the mean of the sample was 18.72 and the median 19.26 (with standard deviation of 2.4) (Figure 1).

The last variable that was analyzed was the ability to clearly express moral beliefs (B score). The min. value for Romanian sample was 0.928 and the max. value was 3.102. The mean was 1.67 , the median was 1.60 (with standard deviation of 0.48 ). For the Polish sample, the min. value was 0.45 and the max. value 2.72. The mean of the sample was 1.46, the median was 1.40 (with standard deviation of 0.49) (Figure 2).

Based on the analysis we conducted, it seems Romanian students have higher EM level and higher B score than Polish students, even though the mean age of Romanians is lower than the one of the Poles. In order to observe if there is a correlation between EM and B level, we conducted a regression analysis, assuming a linear correlation. Additionally, we add gender and age to see if they have an influence on B level (Table 3).

\begin{tabular}{|c|c|c|c|c|}
\hline \multirow{3}{*}{ Independent variable } & \multirow{2}{*}{\multicolumn{2}{|c|}{$\begin{array}{c}\text { Romanian sample } \\
\begin{array}{c}\text { Dependent variable Romanian } \\
\text { bioethics score }\end{array}\end{array}$}} & \multicolumn{2}{|c|}{ Polish sample } \\
\hline & & & \multicolumn{2}{|c|}{$\begin{array}{l}\text { Dependent variable Polish } \\
\text { bioethics score }\end{array}$} \\
\hline & Value & $P$ value & Value & $\mathrm{P}$ value \\
\hline Constant & 1.062 & 0.538 & 2.195 & $0.029 * *$ \\
\hline Age & -0.005 & 0.950 & 0.011 & 0.800 \\
\hline Gender & 0.311 & $0.034 * *$ & -0.315 & $0.041 * *$ \\
\hline Emotional Maturity & 0.026 & 0.429 & -0.039 & 0.174 \\
\hline R squared & $9.8 \%$ & & $11.5 \%$ & \\
\hline F test & 1.768 & 0.166 & 2.000 & 0.127 \\
\hline
\end{tabular}

Based on Table 3, several conclusions can be extracted. Before interpreting the results, we have to emphasize that the model includes qualitative data that was quantified as quantitative. Thus, a part of the information is lost as quantitative information cannot encounter all qualitative features that both B and EM have. Moreover, considering that the analysis conducted is quantitative and on a small sample, we are rather interested in the sign of the correlation than their significance level. The results presented in Tab. 3 reveal a different behavior and attitude for Romanian and Polish students. Considering the age variable, it seems Romanian students decrease their B score as their age increases, while the Polish B score increases with age. On the other hand, we can observe an opposite relationship when gender is taken into consideration. Thus, Romanian women seem to have higher B than Romanian men, while for Polish women this indicator is smaller than the one Polish men have. Surprisingly, EM is related in different terms with B level. While for Romanians there is a positive correlation, meaning an increase in the EM level generates an increase in B level, for Poles, a negative correlation can be observed. Regarding the statistical significance of the generated relations, the models could be considered valid if we assume a risk of $16.6 \%$ of rejecting the null hypothesis that all coefficients are not different from zero. We can assume this as the number of observation included into the analysis is small, so our coefficients can be biased. Thus, the created model can be considered as valid. If we assume the classical approach of statistically significant, we emphasize that gender influence is relevant at a $5 \%$ significance level. Moreover, for EM the relevance of the relationship is found statistically significant for the Polish sample when a risk of $17.7 \%$ is taken into account.

Table 4: The influence of gender on bioethics level both for Romanian and Polish students

\begin{tabular}{|l|c|c|c|c|c|c|c|c|}
\hline \multirow{3}{*}{$\begin{array}{c}\text { Independent } \\
\text { variable }\end{array}$} & \multicolumn{3}{|c|}{ Romanian sample } & \multicolumn{3}{c|}{ Polish sample } \\
\cline { 2 - 9 } & \multicolumn{2}{|c|}{ Dependent variable: Romanian bioethics score } & \multicolumn{2}{c|}{ Dependent variable: Polish bioethics score } \\
\cline { 2 - 9 } & Value & P value & Value & P value & Value & P value & Value & P value \\
\hline Constant & 2.165 & 0.319 & -2.016 & 0.433 & 1.190 & 0.323 & 3.797 & $0.063^{*}$ \\
\hline Age & -0.46 & 0.648 & 0.152 & 0.208 & 0.036 & 0.471 & -0.058 & 0.530 \\
\hline EM & 0.028 & 0.497 & 0.021 & 0.668 & -0.031 & 0.377 & -0.050 & 0.374 \\
\hline R squared & $1.9 \%$ & & $13 \%$ & & $3.8 \%$ & & $12.2 \%$ & 0.457 \\
\hline F test & 0.33 & 0.721 & 0.973 & 0.404 & 0.630 & 0.539 & 0.837 & 0.45 \\
\hline Source: Author & \multicolumn{7}{|c|}{ Male } \\
\hline
\end{tabular}


As the results are referring to the entire sample, we considered it is interesting to see if the correlation in terms of significance is similar for women and men, Romanians and Polish, or if the gender has an influence on the overall results. Thus, regression was re-estimated considering the respondents' gender (Table 4).

From Table 4, it can be seen that the overall influence of age on B score is based on the women's influence, so gender affects the general results. In both Romanian and Polish samples women outnumber men twice. On the other hand, it can be observed, for both men and Romanian women there is a positive relationship between EM and B score, meaning that higher is EM, the higher is B. In comparison, Poland registers a negative relationship between EM and B for both men and women. In terms of statistical significance, the models are not valid due to the lower dimension of the sample taken into account. If we take a look, at the value of the coefficients, even though they are not statistically significant, we can assume that women impact on B is higher than the one of men. The results could also reveal women have higher EM than men. In order to see if women have indeed higher EM, we computed the mean values, the minimum and the maximum values and we provided them in a comparison analysis. Results are presented in Table 5.

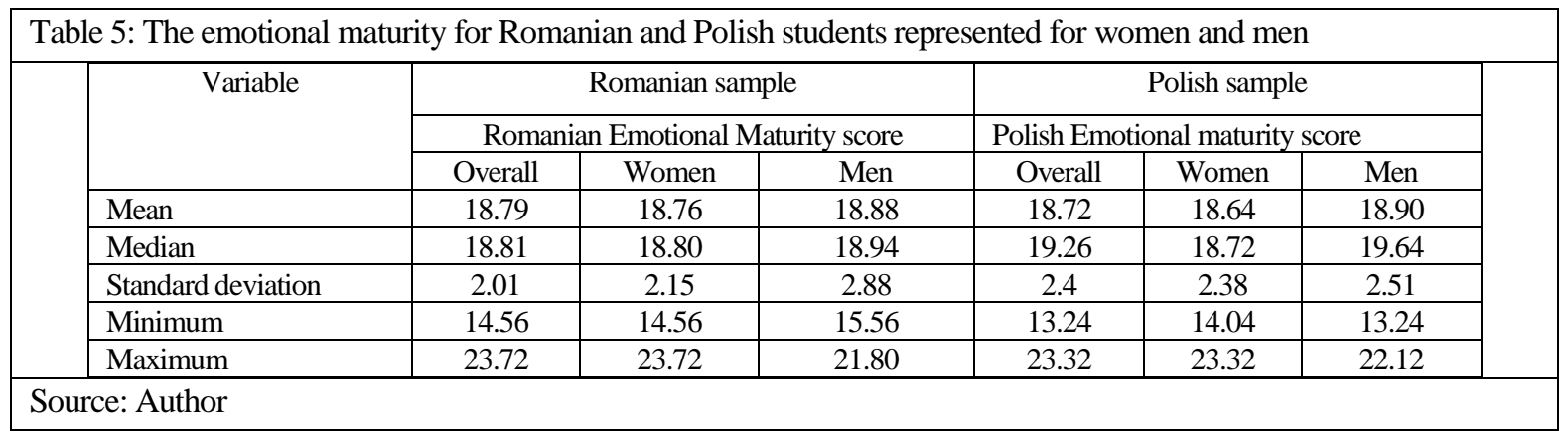

From Table 5, it can be seen the minimum EM score is smaller for Romanian women compared with Romanian men, while in Poland the relationship is different as the minimum EM score is found for Polish men not women. In terms of maximum value, it is achieved both in Romania and Poland by women. Regarding mean and median, we observed the values are smaller for women than men, but we have to take into consideration the size of women sample is twice the size of the men's. When standard deviation is analyzed, we observe women have lower standard deviation than men. Based on these computations we can assume women's data are clustered closely around the mean.

\section{Discussion}

The results reveal different correlations for both countries but are complementary to the studies of other authors, such as Pfeffer (1990) and WHO (2004), arguing that Romanians become aware of their sexuality relatively early. The authors however, did not try to explain this phenomena in emotions or morality related terms. Another possible explanation for the age-bioethics disproportionality can be pointed out by Pfeffer (1990) and Stephenson et al. (1992), the higher social permissiveness towards bioethical issues in Romania than in Poland which is best reflected by the situation described by Pfeffer (1990), that after the National Salvation Front's legalization of contraception and abortion Romanian hospitals were sieged by women. One of the hospitals in Bucharest was visited by 200 women demanding an abortion in a four-hour period. On the contrary, such thing never happened in Poland. Also, Polish students, due to more conservative environment, are being taught about their sexuality later. Hence, we can conclude that their emotional reaction is triggered just while Romanian students enter in the next (rational) stage of bioethical considerations.

The overall influence of age on B score is based on the women's influence, so it can be assumed that gender affects the general results, especially because in both Romanian and Polish samples women outnumbered men. This does not mean however, that the women's result took dominance which was reflected by the fact that Romanian women seem to have higher bioethical values than men, while for the Polish women this indicator is significantly smaller than the one that men have. This result, or at least its second part, contradicts the presuppositions of gender and feminist discourses that bioethical cases concern women in higher degree than men and reveal a gap in bioethical studies which usually neglect e.g., men's role in family planning. It also let us presuppose that these and other general concepts, such as health, are not gender (Płotka, 2015) but culture-related. The latter assumption is also supported by the fact that for both men and women in Romania there is a positive EM-B relationship, meaning that a higher EM score generated a higher B score. In comparison with this, in Poland there is a negative EM-B relationship (the higher EM is, the lower B 
becomes) also for both men and women. Eventually, these results demonstrate the $\mathrm{H} 2$, that women have higher emotional maturity than men, but falsify its assumed meaning that this very fact makes women's impact on B score bigger.

What is surprising in the context of this study's pre-initial question "does increasing the public awareness of bioethical issues through education require modernization?" and the first hypothesis (H1) of this study is the result that EM is differently related to bioethical score level amongst Romanian and Polish students.

While for Romanians there is a positive correlation between EM and B level, meaning that an increase in the EM level generates an increase in B level, for Poles a negative correlation can be observed, meaning that higher is the EM level, the lower is the B level. In other words, emphasizing the role of emotions for teaching bioethics in Romania could improve students' comprehension and awareness while in Poland it could result in the opposite effect. In the context of evaluating Macer's Moral Games for Teaching Bioethics it shows that its educational impact may differ according to the social and cultural environment in which it was used. In much broader sense, this study deploys an argument against Beauchamp and Childress (2001) idea of global bioethics and any bioethics unification projects as its derivatives, while the argument resides within the statement that the first hypothesis of this research is validated only for the Romanian sample.

There are limitations to this study that need to be challenged. The first one is the small sample size which is also a reason for the low statistical significance of the obtained results. Another limitation is the reductive nature of quantifying the qualitative data. Another element is political culture. Despite that Polish and Romanian cultures seem to be similar, they may differ in substantive aspects. That creates a field for conducting broader interdisciplinary studies. Nevertheless, based on this study we discern a need for further intercultural research with the emphasis on the qualitative differences in understanding bioethics and its problems; and for interdisciplinary studies involving bigger samples of respondents and more advanced methodology.

\section{Acknowledgements}

Hereby we would like to thank students from the Faculty of Management, the Faculty of Marketing and the Faculty of Finance, Insurance, Banks and Stock Exchange, Bucharest University of Economic Studies (Romania) and students from the Faculty of Political Science and International Studies, Nicolaus Copernicus University (Poland), for taking a part in our study.

\section{References}

Arbune, M., Potârnichie, O., Păsărin, L., Mârţu, S. (2014). Ethical Dilemma Related to Therapeutic Nonadherence of Hiv Youth. Revista Română de Bioetică, 12(1), 72-79.

Athota, V.S., O'Connor, P.J., Jackson, C. (2009). The Role of Emotional Intelligence and Personality in Moral Reasoning. In Hicks, R.E. (Ed.). Personality and Individual Differences: Current Directions. Bowen Hills: Australian Academic Press.

Beauchamp, T.L., Childress J.F. (2001). Principles of Biomedical Ethics, Oxford: Oxford University Press.

Craşovan, D.I., Ioviță, C. (2013). Cognitive and Emotional Factors Related to High School Aggressive Behaviour. Retrieved from https://www.academia.edu/20120673/Cognitive_and_emotional_factors_related_to_high_school_aggressive_behaviour

Fernandez-Berrocal, P., Extremera, N. (2005). About Emotional Intelligence and Moral Decisions. Behavioral and Brain Sciences, 28(4), 548-549.

Fukuyama, F. (2002). Our Posthuman Future. Consequences of the Biotechnology Revolution. New York: Farrar, Straus and Giroux Press.

Kass, L. (1997). The Wisdom of Repugnance. The New Republic, 2, 17-26.

Keidar, D. (2005). Classroom Communication. Use of Emotional Intelligence and Non-Verbal Communication in Ethics Education at Medical Schools. Israel, Haifa: Israel National Commission for UNESCO.

Kornilova, T.V., Chigrinova, I.A. (2014). Personal Values, Moral Development, and Emotional Intelligence in the Regulation of Choice in Situations that Involve Interpersonal Interactions. Psychology. Journal of the Higher School of Economics, 11(4), 56-74.

Macer, D.R.J. (1998). Bioethics is Love of Life: An Alternative Textbook. Christchurch, N.Z.: Eubios Ethics Institute.

Macer, D.R.J. (2008). Moral Games for Teaching Bioethics. Israel, Haifa: Israel National Commision for UNESCO.

Mărghitan, A.L., Craşovan, D.I. (2014). Aspects Regarding the Conative Self-Regulation in Academic Learning. Research Journal of Agricultural Science, 46(2), 152-155.

Moldovan, O.D. (2014). The Impact of Divorce on Women. Agora Psycho-Pragmatica, 8(2), 5-28.

Parsa, N.A. (2015). The Influence of Emotional Intelligence on Morality Judgement Among Primary School Students in Tehran [Master Thesis]. Malaysia: Universiti Sains Malaysia.

Pfeffer, N. (1990). Fertility Control in Hungary, Romania and Poland. Critical Public Health, 1(2), 16-19. 
Płotka, B. (2015). Gender, Health and Plastic Surgeries. In Gardiner, K., Pfister, S.V. (Ed.). Past and Present: Perspectives on Gender and Love. Oxford: Inter-Disciplinary Press.

Roache, R., Clarks S. (2009). Bioconservatism, Bioliberalism, and the Wisdom of Reflecting on Repugnance. Monash Bioethics Review, 28(1), 4.1-4.21.

Ronald, D. (1986). Law's Empire, Cambridge: Harvard University Press.

Salovey, P., Mayer, J.D. (1990). Emotional Intelligence. Imagination, Cognition, \& Personality, 9, 185-211.

Sivanathan, N., Fekken, G. (2001). Emotional Intelligence, Moral Reasoning and Transformational Leadership. Leadership \& Organization Development Journal, 23(4), 198-204.

Stephenson, P., Wagner, M., Badea, M., Serbanescu, F. (1992). Commentary: The Public Health Consequences of Restricted Induced Abortion - Lessons from Romania. Public Health Policy Forum, 82(10), 1328-1331.

WHO's Abortion and Contraception in Romania: A Strategic Assessment of Policy, Programme and Research Issues (2004). Retrieved from http://apps.who.int/iris/bitstream/10665/43113/1/9739953166.pdf

World Value Survey (2017). Retrieved from http://www.worldvaluessurvey.org/images/Culture_Map_2017_conclusive.png 